\title{
Model identification of linear parameter varying aircraft systems
}

\author{
Atsushi Fujimori, Lennart Ljung \\ Division of Automatic Control \\ E-mail: tmafuji@ipc.shizuoka.ac.jp, ljung@isy.liu.se
}

14th June 2007

Report no.: LiTH-ISY-R-2789

Accepted for publication in Proc. IMechE Vol 220 Part G: Journal Aerospace Engineering

Address:

Department of Electrical Engineering

Linköpings universitet

SE-581 83 Linköping, Sweden

WWW: http://WwW. control.isy.liu.se

AUTOMATIC CONTROL

REGLERTEKNIK

LINKÖPINGS UNIVERSITET

Technical reports from the Automatic Control group in Linköping are available from http://www. control.isy.liu.se/publications. 


\begin{abstract}
This article presents parameter estimation of continuous-time polytopic models for a linear parameter varying (LPV) system. The prediction error method of linear time invariant (LTI) models is modified for polytopic models. The modified prediction error method is applied to an LPV aircraft system whose varying parameter is the flight velocity and model parameters are the stability and control derivatives (SCDs). In an identification simulation, the polytopic model is more suitable for expressing the behaviours of the LPV aircraft than the LTI model regarding time and frequency responses. The SCDs of the initial polytopic model are adjusted to fit the model output dta obtained from the LPV aircraft system.
\end{abstract}

Keywords: identification 


\title{
Model identification of linear parameter varying aircraft systems
}

\author{
A Fujimori $^{1 *}$ and L Ljung ${ }^{2}$ \\ ${ }^{1}$ Department of Mechanical Engineering, Shizuoka University, Shizuoka, Hamamatsu, Japan \\ ${ }^{2}$ Department of Electrical Engineering, Linköping University, Linköping, Sweden
}

The manuscript was received on 21 June 2005 and was accepted after revision for publication on 24 February 2006.

DOI: 10.1243/09544100JAERO28

\begin{abstract}
This article presents a parameter estimation of continuous-time polytopic models for a linear parameter varying (LPV) system. The prediction error method of linear time invariant (LTI) models is modified for polytopic models. The modified prediction error method is applied to an LPV aircraft system whose varying parameter is the flight velocity and model parameters are the stability and control derivatives (SCDs). In an identification simulation, the polytopic model is more suitable for expressing the behaviours of the LPV aircraft system than the LTI model from the viewpoints of the time and the frequency responses. The SCDs of the initial polytopic model are adjusted so as to fit the model output to the output data obtained from the LPV aircraft system.
\end{abstract}

Keywords: polytopic model, system identification, prediction error method, aircraft model

\section{INTRODUCTION}

Linearized equations of aircraft are regarded as linear time invariant (LTI) systems if the altitude and the flight velocity are constant, but as linear parameter varying (LPV) systems if they are varying. Recently, a number of flight control designs using gain scheduling techniques in which the aircraft is treated as an LPV system have been proposed $[1,2]$. In those gain scheduling designs, the LPV system is expressed or sometimes approximated by a polytopic model which is constructed by a linear combination of multiple LTI models at the vertices of the operating region [1]. Then, the control design constraints are expressed by a finite number of linear matrix inequalities (LMIs) [3]. A gain scheduling controller is obtained by solving the LMIs numerically. Unfortunately, in general, it is not always possible to exactly transform an LPV system into a polytopic model. It depends on the structure of the LPV system [4]. One of the simplest

* Corresponding author: Department of Mechanical Engineering, Shizuoka University, 3-5-1 Johoku, Shizuoka, Hamamatsu 4328561, Japan.email: tmafuji@ipc.shizuoka.ac.jp ways for constructing a polytopic model is that multiple operating points are chosen on the range of the varying parameters, an LTI model is obtained at each operating point, and a polytopic model is then constructed by interpolating between the operating points [1]. However, the polytopic model obtained may contain model errors, although it depends on the interpolating function. The model errors cause conservative controllers and deteriorative control performance. Therefore, it is worthwhile to obtain a suitable polytopic model for the original LPV system.

The purpose of this article is to find a desirable polytopic model for an LPV system by system identification. The system identification technique used in this article is the prediction error method based on the quadratic criterion of the prediction error in the time domain [5]. This article modifies the standard prediction error method for polytopic models. The modified prediction error method is applied to an LPV aircraft system for longitudinal motion whose varying parameter is the flight velocity. The estimated polytopic model is evaluated by the time response and the $\nu$-gap metric, which is a criterion associated with the model uncertainty $[6]$. 


\section{POLYTOPIC MODEL AND OBJECTIVE OF PARAMETER ESTIMATION}

Let us consider a continuous-time LPV system given by

$$
\begin{aligned}
\frac{\mathrm{d} \boldsymbol{x}_{\mathrm{pv}}(t)}{\mathrm{d} t} & =\mathbf{A}_{\mathrm{lpv}}(\tau(t), \boldsymbol{\xi}(\tau)) \boldsymbol{x}_{\mathrm{lpv}}(t)+\mathbf{B}_{\mathrm{lpv}}(\tau(t), \xi(\tau)) \mathbf{v}(t) \\
\mathbf{y}_{\mathrm{lpv}}(t) & =\mathbf{C}_{\mathrm{lpv}}(\tau(t), \boldsymbol{\xi}(\tau)) \boldsymbol{x}_{\mathrm{lpv}}(t)+\mathbf{D}_{\mathrm{lpv}}(\tau(t), \boldsymbol{\xi}(\tau)) \mathbf{v}(t)
\end{aligned}
$$

where $x_{\mathrm{lpv}}(t), \boldsymbol{y}_{\mathrm{lpv}}(t)$, and $\boldsymbol{v}(t)$ are, respectively, the $\boldsymbol{n}_{x}$-dimensional state, $n_{y}$-dimensional output, and $\boldsymbol{n}_{v}$-dimensional input vectors. $\tau(t)$ is a measurable varying parameter with respect to time $t . \xi(\tau)$ is the $p$-dimensional model parameter vector which is varying with respect to $\tau$. As one of simple approximations of the LPV system (1), this article considers an interpolative polytopic model

$$
\begin{aligned}
\frac{d \boldsymbol{x}(t)}{d t} & =\mathbf{A}_{\text {poly }}(\tau, \boldsymbol{\eta}) \boldsymbol{x}(t)+\mathbf{B}_{\text {poly }}(\tau, \boldsymbol{\eta}) \boldsymbol{v}(t) \\
\boldsymbol{y}(t) & =\mathbf{C}_{\text {poly }}(\tau, \boldsymbol{\eta}) \boldsymbol{x}(t)+\boldsymbol{D}_{\text {poly }}(\tau, \boldsymbol{\eta}) \boldsymbol{v}(t)
\end{aligned}
$$

where $\boldsymbol{x}(t)$ and $\boldsymbol{y}(t)$ are the $\boldsymbol{n}_{x}$-dimensional state and $\mathbf{n}_{y}$-dimensional output vectors of the polytopic model. The matrices of the polytopic model (2) are given as follows

$$
\begin{aligned}
& \mathbf{A}_{\text {poly }}(\tau, \boldsymbol{\eta})=\sum_{i=1}^{r} w_{i}(\tau) \mathbf{A}_{i}\left(\boldsymbol{\xi}_{i}\right) \\
& \mathbf{B}_{\text {poly }}(\tau, \boldsymbol{\eta})=\sum_{i=1}^{r} w_{i}(\tau) \mathbf{B}_{i}\left(\boldsymbol{\xi}_{i}\right) \\
& \mathbf{C}_{\text {poly }}(\tau, \boldsymbol{\eta})=\sum_{i=1}^{r} w_{i}(\tau) \mathbf{C}_{i}\left(\boldsymbol{\xi}_{i}\right) \\
& \mathbf{D}_{\text {poly }}(\tau, \boldsymbol{\eta})=\sum_{i=1}^{r} w_{i}(\tau) \mathbf{D}_{i}\left(\boldsymbol{\xi}_{i}\right)
\end{aligned}
$$

where

$$
\begin{aligned}
& \mathbf{A}_{i}\left(\boldsymbol{\xi}_{i}\right) \triangleq \mathbf{A}_{\mathrm{lpv}}\left(\tau_{i}, \boldsymbol{\xi}_{i}\right), \quad \mathbf{B}_{i}\left(\boldsymbol{\xi}_{i}\right) \triangleq \mathbf{B}_{\mathrm{lpv}}\left(\tau_{i}, \boldsymbol{\xi}_{i}\right) \\
& \mathbf{C}_{i}\left(\boldsymbol{\xi}_{i}\right) \triangleq \mathbf{C}_{\mathrm{lpv}}\left(\tau_{i}, \boldsymbol{\xi}_{i}\right), \quad \mathbf{D}_{i}\left(\boldsymbol{\xi}_{i}\right) \triangleq \mathbf{D}_{\mathrm{lpv}}\left(\tau_{i}, \boldsymbol{\xi}_{i}\right) \\
& \boldsymbol{\xi}_{i} \triangleq \boldsymbol{\xi}\left(\tau_{i}\right), \quad i=1, \ldots, r \\
& \boldsymbol{\eta} \triangleq\left[\xi_{1}^{\mathrm{T}} \ldots \boldsymbol{\xi}_{r}^{\mathrm{T}}\right]^{\mathrm{T}}
\end{aligned}
$$

$\tau_{i}(i=1, \ldots, r)$ are called the operating points which are chosen on the range of the varying parameter. $\mathbf{A}_{i}\left(\boldsymbol{\xi}_{i}\right), \mathbf{B}_{i}\left(\boldsymbol{\xi}_{i}\right), \mathbf{C}_{i}\left(\boldsymbol{\xi}_{i}\right)$, and $\mathbf{D}_{i}\left(\boldsymbol{\xi}_{i}\right)$ are constant matrices with the $i$ th model parameter vector $\xi_{i}$ at the $i$ th operating point. The set of matrices $\left(\mathbf{A}_{i}, \mathbf{B}_{i}, \mathbf{C}_{i}, \mathbf{D}_{i}\right)$ is therefore called the $i$ th local LTI model in this article. $\boldsymbol{\eta}$ is defined as the collected model parameter vector whose size is $p \times r . w_{i}(\tau), i=1, \ldots, r$, are the weighting functions which satisfy the following relations

$$
w_{i}(\tau) \geqslant 0 \quad \forall i, \quad \sum_{i=1}^{r} w_{i}(\tau)=1
$$

The polytopic model (2) is constructed by interpolating between $r$ operating points with the weighting functions satisfying equation (6). When $r=1$, $w_{1}(\tau)=1$, i.e. equation (2) becomes an LTI model.

A number of modelling for gain scheduling control have been presented $[\mathbf{1}, \mathbf{4}, \mathbf{7}, \mathbf{8}]$. An advantage of equation (2) is that the construction of polytopic models is easy not only for LPV systems but also for non-linear systems whose reference trajectory is given in advance $[\mathbf{9}, \mathbf{1 0}]$. In particular, when the varying parameter is complicatedly included in the LPV system, it may be hard to find a polytopic model which is equivalent to the LPV system. However, the polytopic model (2) may include errors and may not be a proper model of the LPV system (1). Because of this, the model parameters of the polytopic model should be modified so that the polytopic model (2) suitably expresses the behaviours of the LPV system (1).

In this article, applying a system identification technique to the polytopic model (2) which was interpolatively constructed, the model parameters are adjusted so as to fit the output of the polytopic model $\boldsymbol{y}(t)$ to that of the LPV system $\boldsymbol{y}_{\mathrm{lpv}}(t)$ as close as possible, i.e. a desirable polytopic model is found using $v(t)$ and $\boldsymbol{y}_{\mathrm{lpv}}(t)$ as identification data.

There are options for constructing the polytopic model (2) associated with the operating points and the weighting functions. In the former, when the number of the operating points is increased, the model error is decreased [9], but the polytopic model becomes more complicated. In the latter, there are a number of candidates for the weighting functions satisfying equation (6). One of the simplest weighting functions is a triangular function whose centre is at the operating point, as shown in Fig. 1. Other weighting functions are introduced in reference [11]. In this article, it is assumed that the

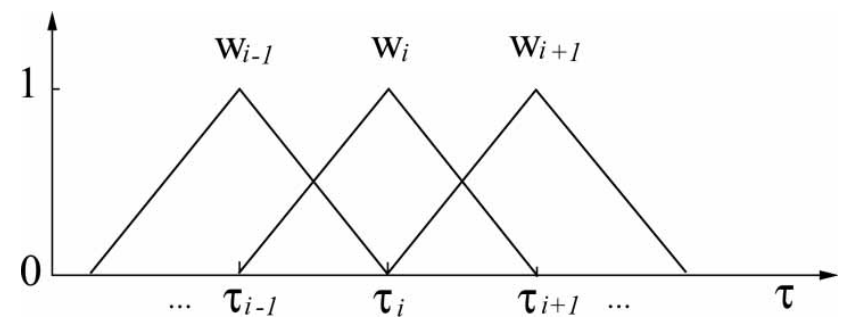

Fig. 1 Triangular interpolative function 
number of the operating points is determined and the weighting functions $w_{i}(\tau), i=1, \cdots, r$, are given in advance.

The polytopic model is one of the blended multiple models $[\mathbf{1 2 - 1 4}]$ in which the varying parameter depends on the input and/or the state. In the polytopic model (2) considered in this article, the varying parameter $\tau(t)$ is independent of these, but depends on time $t$.

\section{PREDICTION ERROR METHOD FOR POLYTOPIC MODEL}

This section shows the computation for parameter estimation in which the prediction error method [5] is modified for polytopic models. Compared with the case of LTI models, there are two novelties in the case of polytopic models: first, number of model parameters to be estimated is proportional to that of chosen operating points. The second is an assumption on the discretization that the varying parameter is frozen for each sampling interval. The discretized predictor and the gradient are discretized piecewise for each sampling. These increase the computational burden on the parameter estimation.

\section{Predictor}

In the prediction error method in the time domain [5], the model parameters are estimated by minimizing a quadratic function of the prediction error, which is the difference between the true and the predicted outputs. A predictor of the model to be estimated is needed to produce the predicted output. In the state-space models, the innovation form [5] is used as a predictor and is essentially the same as an observer of the state-space model. A continuoustime predictor for the polytopic model (2) is given by

$$
\begin{aligned}
\frac{\mathrm{d} \hat{\boldsymbol{x}}(t, \boldsymbol{\eta})}{\mathrm{d} t}= & \mathbf{A}_{\mathrm{poly}}(\tau, \boldsymbol{\eta}) \hat{\boldsymbol{x}}(t, \boldsymbol{\eta})+\mathbf{B}_{\mathrm{poly}}(\tau, \boldsymbol{\eta}) \boldsymbol{v}(t) \\
& +\mathbf{K}_{\mathrm{poly}}(\tau, \boldsymbol{\eta})\left(\boldsymbol{y}_{\mathrm{lpv}}(t)-\hat{\boldsymbol{y}}(t, \boldsymbol{\eta})\right) \\
\hat{\boldsymbol{y}}(t, \boldsymbol{\eta})= & \mathbf{C}_{\mathrm{poly}}(\tau, \boldsymbol{\eta}) \hat{\boldsymbol{x}}(t, \boldsymbol{\eta})+\mathbf{D}_{\mathrm{poly}}(\tau, \boldsymbol{\eta}) \boldsymbol{v}(t)
\end{aligned}
$$

where $\hat{\boldsymbol{x}}(t, \boldsymbol{\eta})$ and $\hat{\boldsymbol{y}}(t, \boldsymbol{\eta})$ are the $\boldsymbol{n}_{x}$-dimensional predicted state and $\boldsymbol{n}_{y}$-dimensional predicted output vectors, respectively. $\mathbf{K}_{\text {poly }}(\tau, \boldsymbol{\eta})$ is a filter gain which is given so that $\mathbf{A}_{\text {poly }}(\tau, \boldsymbol{\eta})-\mathbf{K}_{\text {poly }}(\tau, \boldsymbol{\eta}) \mathbf{C}_{\text {poly }}(\tau, \boldsymbol{\eta})$ is a stable matrix. Similar to equation (3), $\mathbf{K}_{\text {poly }}(\tau, \boldsymbol{\eta})$ is given by the following polytopic form

$$
\mathbf{K}_{\mathrm{poly}}(\tau, \boldsymbol{\eta})=\sum_{i=1}^{r} \omega_{i}(\tau) K_{i}\left(\boldsymbol{\xi}_{i}\right)
$$

Denoting the input and the output of the LPV system as

$$
z(t) \triangleq\left[y_{1 \mathrm{pv}}^{\mathrm{T}}(t) \quad \boldsymbol{v}^{\mathrm{T}}(t)\right]^{\mathrm{T}}
$$

equation (7) is compactly written as

$$
\begin{aligned}
\frac{\mathrm{d} \hat{\boldsymbol{x}}(t, \boldsymbol{\eta})}{\mathrm{d} t} & =\mathbf{F}_{\mathrm{poly}}(\tau, \boldsymbol{\eta}) \hat{\boldsymbol{x}}(t, \boldsymbol{\eta})+\mathbf{G}_{\mathrm{poly}}(\tau, \boldsymbol{\eta}) z(t) \\
\hat{\boldsymbol{y}}(t, \boldsymbol{\eta}) & =\mathbf{C}_{\mathrm{poly}}(\tau, \boldsymbol{\eta}) \hat{\boldsymbol{x}}(t, \boldsymbol{\eta})+\mathbf{H}_{\mathrm{poly}}(\tau, \boldsymbol{\eta}) z(t)
\end{aligned}
$$

where

$$
\begin{aligned}
& \mathbf{F}_{\text {poly }}(\tau, \boldsymbol{\eta}) \triangleq \mathbf{A}_{\text {poly }}(\tau, \boldsymbol{\eta})-\mathbf{K}_{\text {poly }}(\tau, \boldsymbol{\eta}) \mathbf{C}_{\text {poly }}(\tau, \boldsymbol{\eta}) \\
& \mathbf{G}_{\text {poly }}(\tau, \boldsymbol{\eta}) \triangleq\left[\mathbf{K}_{\text {poly }}(\tau, \boldsymbol{\eta}) \quad \mathbf{B}_{\text {poly }}(\tau, \boldsymbol{\eta})\right. \\
& \left.-\mathbf{K}_{\text {poly }}(\tau, \boldsymbol{\eta}) \mathbf{D}_{\text {poly }}(\tau, \boldsymbol{\eta})\right] \\
& \mathbf{H}_{\text {poly }}(\tau, \boldsymbol{\eta}) \triangleq\left[0 \quad \mathbf{D}_{\text {poly }}(\tau, \boldsymbol{\eta})\right]
\end{aligned}
$$

\subsection{Discretization}

As the data used for parameter estimation are sampled from the LPV system with a constant time interval $T$, a discrete representation of the predictor is needed for computing the estimates of the parameter. When $T$ is chosen as a sufficiently small value compared with the change of $\tau(t)$, it is valid that $\tau(t)$ is constant for each sampling interval; that is

$$
\tau(t)=\tau(k T), \quad k T \leqslant t<k T+T
$$

Then,

$\mathbf{F}_{\text {poly }}(\tau(k T), \boldsymbol{\eta}), \mathbf{G}_{\text {poly }}(\tau(k T), \boldsymbol{\eta}), \mathbf{C}_{\text {poly }}(\tau(k T), \boldsymbol{\eta}), \quad$ and $\mathbf{H}_{\text {poly }}(\tau(k T), \boldsymbol{\eta})$ are constant with fixed $\boldsymbol{\eta}$ when $k T \leqslant t<k T+T$. Applying the zero-order hold discretization to predictor (10), the following piecewise discrete-time predictor is obtained

$$
\begin{aligned}
\hat{\boldsymbol{x}}(k T+T, \boldsymbol{\eta})= & \mathbf{F}(\tau(k T), \boldsymbol{\eta}) \boldsymbol{x}(k T, \boldsymbol{\eta}) \\
& +\mathbf{G}(\tau(k T), \boldsymbol{\eta}) z(k T) \\
\hat{\boldsymbol{y}}(k T, \boldsymbol{\eta})= & \mathbf{C}(\tau(k T), \boldsymbol{\eta}) \boldsymbol{x}(k T, \boldsymbol{\eta}) \\
& +\mathbf{H}(\tau(k T), \boldsymbol{\eta}) z(k T)
\end{aligned}
$$

where

$$
\begin{aligned}
& \mathbf{F}(\tau(k T), \boldsymbol{\eta}) \triangleq \mathrm{e}^{\mathbf{F}_{\mathrm{poly}}(\tau(k T), \boldsymbol{\eta}) T} \\
& \mathbf{G}(\tau(k T), \boldsymbol{\eta}) \triangleq \int_{0}^{T} \mathrm{e}^{\mathbf{F}_{\mathrm{poly}}(\tau(k T), \boldsymbol{\eta}) s} \mathrm{~d} s \mathbf{G}_{\mathrm{poly}}(\tau(k T), \boldsymbol{\eta}) \\
& \mathbf{C}(\tau(k T), \boldsymbol{\eta}) \triangleq \mathbf{C}_{\mathrm{poly}}(\tau(k T), \boldsymbol{\eta}) \\
& \mathbf{H}(\tau(k T), \boldsymbol{\eta}) \triangleq \mathbf{H}_{\mathrm{poly}}(\tau(k T), \boldsymbol{\eta})
\end{aligned}
$$


Equation (12) is valid during each sampling interval. The discretization of the predictor has to be done at each sampling.

\subsection{Quadratic function and the Gauss-Newton method}

A quadratic function of the prediction error is given in the prediction error method [5]. The estimate of the parameter is then found so that the quadratic function is minimized. This article adopts the Gauss-Newton method for the minimization. Let $N$ be the number of the sampled data. The data set $Z^{N}$ is defined as

$$
\mathbf{Z}^{N} \triangleq\left[\mathbf{z}^{\mathrm{T}}(T) \cdots \mathbf{z}^{\mathrm{T}}(N T)\right]^{\mathrm{T}}
$$

A quadratic function to be minimized is given by

$$
J_{N}\left(\boldsymbol{\eta}, \mathbf{Z}^{N}\right) \triangleq \frac{1}{N} \sum_{k=1}^{N} \frac{1}{2} \mathrm{e}^{\mathrm{T}}(k T, \boldsymbol{\eta}) \boldsymbol{e}(k T, \boldsymbol{\eta})
$$

where $\boldsymbol{e}(k T, \boldsymbol{\eta})$ is the prediction error vector defined as

$$
\boldsymbol{e}(k T, \boldsymbol{\eta}) \triangleq \boldsymbol{y}_{\mathrm{lpv}}(k T)-\hat{\boldsymbol{y}}(k T, \boldsymbol{\eta})
$$

Then, the collected model parameter vector is estimated as

$$
\hat{\boldsymbol{\eta}}=\arg \min _{\boldsymbol{\eta}} J_{N}\left(\boldsymbol{\eta}, \mathbf{Z}^{N}\right)
$$

As $\boldsymbol{\eta}$ is implicitly included in the prediction error $\boldsymbol{e}(k T, \boldsymbol{\eta}), J_{N}$ cannot be explicitly expressed with respect to $\boldsymbol{\eta}$. The minimization of $J_{N}$ is then done numerically by an iterative calculation. In this article, the Gauss-Newton method is used to search $\boldsymbol{\eta}$ minimizing $J_{N}$. Letting the superscript ( $i$ ) be the iteration number, the collected model parameter vector is updated by

$$
\hat{\boldsymbol{\eta}}^{(i+1)}=\hat{\boldsymbol{\eta}}^{(i)}-\mu^{(i)}\left[H_{N}^{(i)}\right]^{-1} J_{N}^{\prime}\left(\hat{\boldsymbol{\eta}}^{(i)}, \mathbf{Z}^{N}\right)
$$

where

$$
\begin{aligned}
J_{N}^{\prime}\left(\hat{\boldsymbol{\eta}}^{(i)}, \mathbf{Z}^{N}\right) & \triangleq-\frac{1}{N} \sum_{k=1}^{N} \psi^{\mathrm{T}}\left(k T, \hat{\boldsymbol{\eta}}^{(i)}\right) \boldsymbol{e}\left(k T, \hat{\boldsymbol{\eta}}^{(i)}\right) \\
H_{N}^{(i)} & \triangleq \frac{1}{N} \sum_{k=1}^{N} \psi^{\mathrm{T}}\left(k T, \hat{\boldsymbol{\eta}}^{(i)}\right) \psi\left(k T, \hat{\boldsymbol{\eta}}^{(i)}\right) \\
\psi\left(k T, \hat{\boldsymbol{\eta}}^{(i)}\right) & \triangleq \frac{\partial \hat{\boldsymbol{y}}\left(k T, \hat{\boldsymbol{\eta}}^{(i)}\right)}{\partial \hat{\boldsymbol{\eta}}^{\mathrm{T}}}
\end{aligned}
$$

$H_{N}^{(i)}$ is called the Hessian and is usually invertible. $\mu^{(i)}$ is the step size and $\psi\left(k T, \hat{\boldsymbol{\eta}}^{(i)}\right)$ is the gradient matrix of the prediction error with respect to $\boldsymbol{\eta}$ and is given in the following section.

\subsection{Gradient}

Let $\boldsymbol{\eta}_{l}$ be the $l$ th element of $\boldsymbol{\eta}$. The gradients $\partial \hat{\boldsymbol{y}}(k T, \boldsymbol{\eta}) / \partial \boldsymbol{\eta}_{l}(l=1, \ldots, p r)$ are obtained as the following piecewise discrete-time state-space representation

$$
\begin{aligned}
& \frac{\partial \hat{\boldsymbol{x}}(k T+T, \boldsymbol{\eta})}{\partial \boldsymbol{\eta}_{l}}=\mathbf{F}(\tau(k T), \boldsymbol{\eta}) \frac{\partial \hat{\boldsymbol{x}}(k T, \boldsymbol{\eta})}{\partial \boldsymbol{\eta}_{l}} \\
& +\left[\begin{array}{ll}
\frac{\partial \mathbf{F}(\tau(k T), \boldsymbol{\eta})}{\partial \boldsymbol{\eta}_{l}} & \frac{\partial \mathbf{G}(\tau(k T), \boldsymbol{\eta})}{\partial \boldsymbol{\eta}_{l}}
\end{array}\right] \\
& \times\left[\begin{array}{c}
\hat{\boldsymbol{x}}(k T, \boldsymbol{\eta}) \\
\mathbf{z}(k T)
\end{array}\right] \\
& \frac{\partial \hat{\boldsymbol{y}}(k T, \boldsymbol{\eta})}{\partial \boldsymbol{\eta}_{l}}=\mathbf{C}(\tau(k T), \boldsymbol{\eta}) \frac{\partial \hat{\boldsymbol{x}}(k T, \boldsymbol{\eta})}{\partial \boldsymbol{\eta}_{l}} \\
& +\left[\begin{array}{ll}
\frac{\partial \mathbf{C}(\tau(k T), \boldsymbol{\eta})}{\partial \boldsymbol{\eta}_{l}} & \frac{\partial \mathbf{H}(\tau(k T), \boldsymbol{\eta})}{\partial \boldsymbol{\eta}_{l}}
\end{array}\right] \\
& \times\left[\begin{array}{c}
\hat{\boldsymbol{x}}(k T, \boldsymbol{\eta}) \\
\mathbf{z}(k T)
\end{array}\right], \quad l=1, \ldots, p r
\end{aligned}
$$

The derivatives of $\mathbf{F}(\tau(k T), \boldsymbol{\eta})$ are numerically obtained as

$$
\begin{aligned}
\frac{\partial \mathbf{F}(\tau(k T), \boldsymbol{\eta})}{\partial \boldsymbol{\eta}_{l}} & \simeq \frac{\mathbf{F}\left(\tau(k T), \boldsymbol{\eta}+\delta \boldsymbol{e}_{l}\right)-\mathbf{F}\left(\tau(k T), \boldsymbol{\eta}-\delta \boldsymbol{e}_{l}\right)}{2 \delta}, \\
l & =1, \ldots, p r
\end{aligned}
$$

where $\boldsymbol{e}_{l}$ is the $p r$-dimensional vector whose $l$ th element is one and others are zeros. $\delta$ is a small positive value.

To estimate the model parameters accurately, the input $\boldsymbol{v}(t)$ and the varying parameter $\tau(t)$ should be given to excite all local LTI models. In particular, $\tau(t)$ has to be varied over the entire range. This means that all the weighting functions $w_{i}(\tau) \quad(i=$ $1, \cdots, r)$ may not be constant. Otherwise, the Hessian $H_{N}^{(i)}$ given by equation (19) may be singular, and therefore, the iterative calculation is stopped. In this situation, some local LTI models are not needed in the polytopic model (3). For example, suppose that $w_{1}(\tau)$ is always constant for all measured data. If $w_{1}(\tau)=0$, the first local LTI model $\left(A_{1}, B_{1}\right.$, $\left.C_{1}, D_{1}\right)$ is not needed because of no contribution to equation (3). If $w_{1}(\tau)=1$, the rest of the local LTI models are not needed. Moreover, if $w_{1}(\tau)$ is a constant in the range of $0<w_{1}(\tau)<1$, equation (6) is written as

$$
\sum_{i=2}^{r} \frac{w_{i}}{1-w_{1}}=1
$$


Replacing $w_{i}$ by $\tilde{w}_{i} \triangleq w_{i} /\left(1-w_{1}\right)$, equation (6) are satisfied. Then, the polytopic model is constructed by $r-1$ local LTI models except $\left(A_{1}, B_{1}, C_{1}, D_{1}\right)$.

\section{IDENTIFICATION SIMULATION OF LPV AIRCRAFT SYSTEM}

In flight control designs for specified flight envelope with respect to the flight velocity and the altitude, the aircraft system is regarded as an LPV system. This section presents an identification simulation of an LPV aircraft system for the longitudinal motion to demonstrate the effectiveness of the proposed identification technique. The model parameters in the aircraft system are the stability and control derivatives (SCDs), which express linear contributions of the perturbed velocities and the angular rates to the aerodynamic forces and moments. The SCDs are varied according to the flight conditions, especially the flight velocity. This section considers an LPV aircraft system whose varying parameter is the flight velocity.

\subsection{LPV aircraft system for longitudinal motion}

In the steady flight, the dynamics of aircraft can be generally divided into two parts: the longitudinal and the lateral motions. This section shows an LPV aircraft system for the longitudinal motion. The longitudinal motion in the continuous-time is expressed as the following linearized equations

$$
\begin{aligned}
& \frac{\mathrm{d} u}{\mathrm{~d} t}-X_{u} u-X_{\alpha} \alpha+g \cos \Theta_{0} \theta=0 \\
& -Z_{u} u+V \frac{\mathrm{d} \alpha}{\mathrm{d} t}-Z_{\alpha} \alpha+\left(V+Z_{q}\right) q \\
& \quad+g \sin \Theta_{0} \theta=Z_{\delta_{\mathrm{e}}} \delta_{\mathrm{e}} \\
& -M_{u} u-M_{\dot{\alpha}} \frac{\mathrm{d} \alpha}{\mathrm{d} t}-M_{\alpha} \alpha+\frac{\mathrm{d} q}{\mathrm{~d} t}-M_{q} q=M_{\delta_{\mathrm{e}}} \delta_{\mathrm{e}} \\
& \frac{\mathrm{d} \theta}{\mathrm{d} t}=q
\end{aligned}
$$

where $u$ is the forward velocity, $\alpha$ the angle of attack, $\theta$ the pitch angle, $q$ the pitch rate, and $\delta_{\mathrm{e}}$ the elevator angle. The notations used in equations (24) are based on the symbols that have been usually used in flight dynamics [15]. The variables denoted by small letters mean the perturbed values. $\Theta_{0}$ is the pitch angle in the steady state. $g$ is the acceleration due to gravity. In equation (24), there are nine stability derivatives: $X_{u}, X_{\alpha}, Z_{u}, Z_{\alpha}, Z_{q}, M_{u}, M_{\alpha}, M_{\dot{\alpha}}$, and $M_{q}$ and two control derivatives: $Z_{\delta_{\mathrm{e}}}$ and $M_{\delta_{\mathrm{e}}}$, which are varied with the flight velocity $V$ and the altitude $H$. As $V$ is more considerably influenced on equation (24) rather than $H$, the varying parameter considered in this article is $\tau(t)=V(t)$ and its range is given by

$$
V_{1} \leqslant V(t) \leqslant V_{2}, \quad V_{1}<V_{2}
$$

Defining $\boldsymbol{x}_{\mathrm{lpv}}(t), \boldsymbol{y}_{\mathrm{lpv}}(t)$, and $\boldsymbol{v}(t)$ as

$$
\boldsymbol{x}_{\mathrm{lpv}}(t) \triangleq\left[\begin{array}{c}
u \\
\theta \\
\alpha \\
q
\end{array}\right], \quad \boldsymbol{y}_{\mathrm{lpv}}(t) \triangleq\left[\begin{array}{c}
u \\
\theta \\
\alpha
\end{array}\right], \quad \boldsymbol{v}(t) \triangleq \delta_{\mathrm{e}}
$$

a continuous-time LPV aircraft system for the longitudinal motion is then written as

$$
\begin{aligned}
\frac{\mathrm{d} \boldsymbol{x}_{\mathrm{lpv}}(t)}{\mathrm{d} t} & =\mathbf{A}_{\mathrm{lpv}}(V, \boldsymbol{\xi}(V)) \boldsymbol{x}_{\mathrm{lpv}}(t)+\boldsymbol{B}_{\mathrm{lpv}}(V, \boldsymbol{\xi}(V)) \boldsymbol{v}(t) \\
\boldsymbol{y}_{\mathrm{lpv}}(t) & =\mathbf{C}_{\mathrm{lpv}} \boldsymbol{x}_{\mathrm{lpv}}(t)+\mathbf{D}_{\mathrm{lpv}} \boldsymbol{v}(t)
\end{aligned}
$$

where matrices in equations (27) are given by

$$
\begin{aligned}
& \mathbf{A}_{\mathrm{lpv}} \triangleq\left[\begin{array}{cc}
X_{u} & -g \cos \Theta_{0} \\
0 & 0 \\
\frac{Z_{u}}{V} & -\frac{g \sin \Theta_{0}}{V} \\
M_{u}+M_{\dot{\alpha}} \frac{Z_{u}}{V} & -M_{\dot{\alpha}} \frac{g \sin \Theta_{0}}{V}
\end{array}\right. \\
& X_{\alpha} \quad 0 \\
& \begin{array}{cc}
0 & 1 \\
\frac{Z_{\alpha}}{V} & 1+\frac{Z_{q}}{V}
\end{array} \\
& \left.M_{\alpha}+M_{\dot{\alpha}} \frac{Z_{\alpha}}{V} \quad M_{q}+M_{\dot{\alpha}}\left(1+\frac{Z_{q}}{V}\right)\right] \\
& \mathbf{B}_{\mathrm{lpv}} \triangleq\left[\begin{array}{c}
0 \\
0 \\
\frac{Z_{\delta_{\mathrm{e}}}}{V} \\
M_{\delta_{\mathrm{e}}}+M_{\dot{\alpha}} \frac{Z_{\delta_{\mathrm{e}}}}{V}
\end{array}\right] \\
& \mathbf{C}_{\mathrm{lpv}}=\left[\begin{array}{llll}
1 & 0 & 0 & 0 \\
0 & 1 & 0 & 0 \\
0 & 0 & 1 & 0
\end{array}\right], \quad \mathbf{D}_{\mathrm{lpv}}=\left[\begin{array}{l}
0 \\
0 \\
0
\end{array}\right]
\end{aligned}
$$

The elements of $\boldsymbol{y}_{\mathrm{lpv}}(t)$ are selected to avoid the over-parameterized estimation [16]. Collecting the SCDs, the model parameter vector of the LPV system is given by

$$
\boldsymbol{\xi}(V)=\left[\begin{array}{llllllll}
X_{u} & X_{\alpha} & Z_{u} & Z_{\alpha} & Z_{q} & M_{u} & M_{\alpha} & M_{\dot{\alpha}} \\
& & & M_{q} & Z_{\delta_{\mathrm{e}}} & M_{\delta_{\mathrm{e}}}
\end{array}\right]^{\mathrm{T}}
$$


A polytopic model of the longitudinal motion of aircraft was constructed as follows: two operating points were chosen at both edges of the flight region (25); that is, $V=V_{1}$ and $V_{2}$. Two local LTI models were obtained. Using the linear interpolation as shown in Fig. 1, matrices of the polytopic model (2) for equation (27) were then constructed as

$$
\begin{aligned}
\mathbf{A}_{\mathrm{poly}}(V, \boldsymbol{\eta}) & =\sum_{i=1}^{2} w_{i}(V) \mathbf{A}_{i}\left(\boldsymbol{\xi}_{i}\right), \quad \mathbf{B}_{\mathrm{poly}}(V, \boldsymbol{\eta}) \\
& =\sum_{i=1}^{2} w_{i}(V) \mathbf{B}_{i}\left(\boldsymbol{\xi}_{i}\right) \\
\mathbf{C}_{\mathrm{poly}} & =\mathbf{C}_{\mathrm{lpv}}, \quad \mathbf{D}_{\mathrm{poly}}=\mathbf{D}_{\mathrm{lpv}} \\
\mathbf{A}_{i}\left(\boldsymbol{\xi}_{i}\right) & =\mathbf{A}_{\mathrm{lpv}}\left(V_{i}, \boldsymbol{\xi}_{i}\right), \quad \mathbf{B}_{i}\left(\boldsymbol{\xi}_{i}\right)=\mathbf{B}_{\mathrm{lpv}}\left(V_{i}, \boldsymbol{\xi}_{i}\right) \\
\boldsymbol{\xi}_{i} \triangleq \boldsymbol{\xi}\left(V_{i}\right), \quad i & =1,2 \\
\boldsymbol{\eta} & =\left[\begin{array}{ll}
\boldsymbol{\xi}_{1}^{\mathrm{T}} & \boldsymbol{\xi}_{2}^{\mathrm{T}}
\end{array}\right]^{\mathrm{T}}
\end{aligned}
$$

As the number of the SCDs was 11 for each local LTI model, the number of the model parameters was then $11 \times 2=22$. The weighting functions were given as

$$
w_{1}(V) \triangleq \frac{V_{2}-V}{V_{2}-V_{1}}, \quad w_{2}(V) \triangleq \frac{V-V_{1}}{V_{2}-V_{1}}
$$

\subsection{Data for parameter estimation}

In the identification simulation, the flight velocity was changed in the range of flight region (25). As an example, it was considered a situation that the flight velocity $V(t)$ was constantly accelerated as

$$
V(t)=V_{1}+a_{\nu} t
$$

where $a_{v}$ is the acceleration. The input data for parameter estimation were given by random binary signals. Using the flight velocity and the random input data, the output data were generated by the LPV aircraft system (27). The SCDs of the LPV aircraft system at each sampling were obtained by an analytical calculation based on the quasi-steady aerodynamic theory. They were given by [15].

$$
\begin{aligned}
X_{u} & =\frac{\rho V S}{2 m}\left(C_{x u}+2 C_{L} \tan \Theta_{0}\right) \\
Z_{u} & =\frac{\rho V S}{2 m}\left(C_{z u}-2 C_{\mathrm{L}}\right) \\
M_{u} & =\frac{\rho V S c}{2 I_{y y}} C_{m u}, \quad X_{\alpha}=\frac{\rho V^{2} S}{2 m} C_{x \alpha} \\
Z_{\alpha} & =\frac{\rho V^{2} S}{2 m} C_{z \alpha}, \quad M_{\alpha}=\frac{\rho V^{2} S c}{2 I_{y y}} C_{m \alpha}, \\
M_{\dot{\alpha}} & =\frac{\rho V S c^{2}}{4 I_{y y}} C_{m \dot{\alpha}} \\
Z_{q} & =\frac{\rho V S c}{4 m} C_{z q}, \quad M_{q}=\frac{\rho V S c^{2}}{4 I_{y y}} C_{m q} \\
Z_{\delta_{\mathrm{e}}} & =\frac{\rho V^{2} S}{2 m} C_{z \delta_{\mathrm{e}}}, \quad M_{\delta_{\mathrm{e}}}=\frac{\rho V^{2} S c}{2 m} C_{m \delta_{\mathrm{e}}}
\end{aligned}
$$

where $m$ is the mass of the aircraft, $S$ the main wing area, $c$ the main wing chord, and $b$ the main wing span. $C_{\mathrm{L}}$ was the lift coefficient. $C_{x u}, C_{m \alpha}$, and so on. were the non-dimensional SCDs and were obtained from the structural parameters of the aircraft [15]. The numerical values of the aircraft were referred from reference [17].

The number of the data was $N=100$. The sampling time was given by $T=0.5 \mathrm{~s}$. The acceleration of the flight velocity in equation (32) was given by $a_{v}=2 \mathrm{~m} / \mathrm{s}^{2}$. The initial state was given by $\boldsymbol{x}_{\mathrm{lpv}}(0)=0$. Using the flight velocity and the data explained earlier, the parameter estimation was

\begin{tabular}{|c|c|c|c|c|c|c|c|c|c|c|c|}
\hline Model & $X_{u}$ & $X_{\alpha}$ & $Z_{u}$ & $Z_{\alpha}$ & $Z_{q}$ & $M_{u}$ & $M_{\alpha}$ & $M_{q}$ & $M_{\dot{\alpha}}$ & $Z_{\delta_{e}}$ & $M_{\delta_{e}}$ \\
\hline Initial $^{\mathrm{a}}$ & -0.0237 & 7.8829 & -0.2423 & -88.877 & -1.6869 & 0 & -5.2892 & -1.0613 & -0.3274 & -5.9923 & -3.7699 \\
\hline Estimated & -0.0452 & 4.4446 & -0.2469 & -85.799 & -19.583 & -0.0743 & -32.227 & 27.776 & -35.420 & -21.065 & -9.6394 \\
\hline
\end{tabular}
done in the cases of the LTI and the polytopic models for the purpose of comparison.

\subsection{Parameter estimation results}

\subsubsection{LTI model case}

Table 1 shows the initial and the estimated SCDs in the case of LTI model, where the initial SCDs were given by equation (33) and the flight velocity was $V=110 \mathrm{~m} / \mathrm{s}$. Figure 2 shows the output responses of the initial and the estimated LTI models. The solid- and the dashed-dotted lines mean the output data and the model output, respectively. The responses of both LTI models were not well fitted to the output data. There was no improvement on

Table 1 Initial and estimated SCDs in the case of LTI model

${ }^{\text {a}} S C D s$ were given by equation (33) where the flight velocity was $V=110 \mathrm{~m} / \mathrm{s}$. 

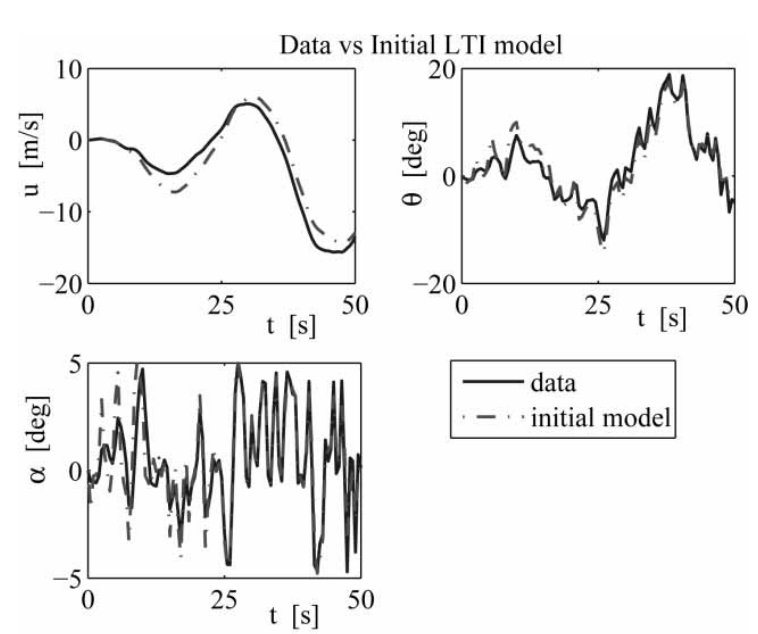

(a) Initial LTI model.
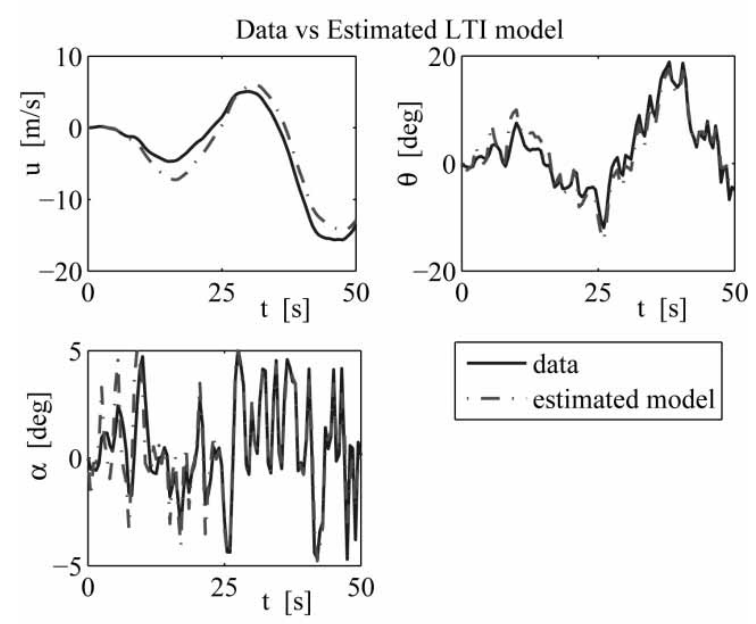

(b) Estimated LTI model.

Fig. 2 Comparison between output data and outputs of initial and estimated LTI models

the estimated LTI model by using the system identification.

The root mean square (r.m.s.) of the prediction error for each output channel is used to evaluate the model output quantitatively. Table 2 shows the r.m.s. of the prediction error for each output channel of the initial and the estimated LTI models, where $\boldsymbol{e}_{u}$, $\boldsymbol{e}_{\alpha}$, and $\boldsymbol{e}_{\theta}$ are the prediction errors of $x$-axis velocity, the angle of attack, and the pitch angle, respectively. Although the estimated LTI model showed smaller

Table 2 R.m.s. of prediction error for each output channel of initial and estimated LTI models

\begin{tabular}{llll}
\hline Model & $\boldsymbol{e}_{u}(\mathrm{~m} / \mathrm{s})$ & $\boldsymbol{e}_{\theta}\left({ }^{\circ}\right)$ & $\boldsymbol{e}_{\alpha}\left({ }^{\circ}\right)$ \\
\hline Initial & 1.7100 & 1.7706 & 0.9801 \\
Estimated & 1.1029 & 1.3528 & 0.9319 \\
\hline
\end{tabular}

values than the initial LTI model, it was not enough to be acceptable.

The $\nu$-gap metric had been introduced in robust control theories associated with the stability margin [6]. It is one of criteria measuring the model error in the frequency domain. It is worth while evaluating the $\nu$-gap metric between the LPV aircraft system and the initial or estimated model. Let $P_{\mathrm{lpv}}(s, V)$ be the transfer function of the LPV system, where the varying parameter is $V$. Let $P_{\mathrm{lti}}(s)$ be that of the initial or the estimated LTI model. The $\nu$-gap metric between $P_{\mathrm{lpv}}(s, V)$ and $P_{\mathrm{lti}}(s)$ is defined as

$$
\delta_{v}\left(P_{\mathrm{lpv}}, P_{\mathrm{lti}}\right) \triangleq \sup _{\omega} \kappa\left(P_{\mathrm{lpv}}(j \omega, V), P_{\mathrm{lti}}(j \omega)\right)
$$

where

$$
\kappa(X, Y) \triangleq \frac{1}{\sigma}\left[\left(I+Y Y^{*}\right)^{1 / 2}(Y-X)\left(I+X X^{*}\right)^{1 / 2}\right]
$$

where $\bar{\sigma} \cdot]$ means the maximum singular value. The range is $\delta_{v} \in[\mathbf{0}, \mathbf{1}]$. A large $\delta_{v}$ means that the model error is large. Figure 3 shows the plots of $\delta_{v}\left(P_{\mathrm{lpv}}, P_{\mathrm{lti}}\right)$, where the solid- and the dashed-dotted lines indicate that $P_{\mathrm{lti}}(s)$ is the estimated and the initial LTI models, respectively. As the SCDs of the initial LTI model were given at $V=110 \mathrm{~m} / \mathrm{s}$, $\delta_{v}\left(P_{\mathrm{lpv}}, P_{\mathrm{lti}}\right)$ whose $P_{\mathrm{lti}}(s)$ was the initial LTI model was zero at $V=110 \mathrm{~m} / \mathrm{s}$. However, it was increased when $V$ was shifted from $V=110 \mathrm{~m} / \mathrm{s}$. In contrast, the minimum of $\delta_{v}\left(P_{\mathrm{lpv}}, P_{\mathrm{lti}}\right)$ whose $P_{\mathrm{lti}}(s)$ was the estimated LTI model was moved to $V \simeq 93 \mathrm{~m} / \mathrm{s}$. Similar to the initial LTI model, it was increased in other flight condition. It was seen that the LTI model was not enough to express the LPV aircraft system from the viewpoints of the time and the frequency responses.

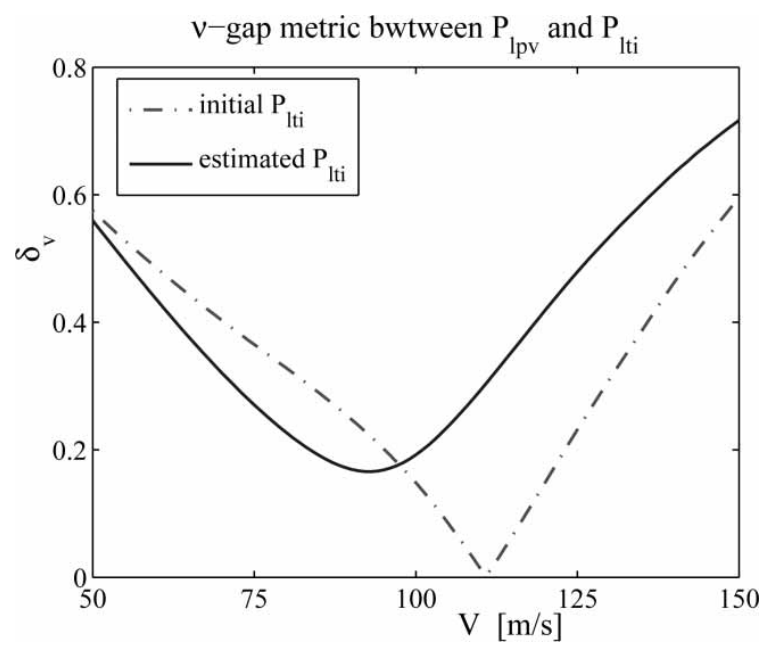

Fig. $3 v$-gap metric between $P_{\mathrm{lpv}}(s, V)$ and $P_{\mathrm{lti}}(s)$ in the flight region, $50 \leqslant V(t) \leqslant 150 \mathrm{~m} / \mathrm{s}$ 
Table 3 Initial and estimated SCDs in the case of polytopic model

\begin{tabular}{|c|c|c|c|c|c|c|c|c|c|c|c|}
\hline Model & $X_{u}$ & $X_{\alpha}$ & $Z_{u}$ & $Z_{\alpha}$ & $Z_{q}$ & $M_{u}$ & $M_{\alpha}$ & $M_{q}$ & $M_{\dot{\alpha}}$ & $Z_{\delta_{e}}$ & $M_{\delta_{e}}$ \\
\hline Initial no. $1^{\mathrm{a}}$ & -0.0108 & 1.6287 & -0.1101 & -18.363 & -0.7668 & 0 & -1.0928 & -0.4824 & -0.1488 & -1.2381 & -77.890 \\
\hline Initial no. $2^{\mathrm{b}}$ & -0.0324 & 14.658 & -0.3303 & -165.27 & -2.3003 & 0 & -9.8353 & -1.4472 & -0.4465 & -11.143 & -7.0101 \\
\hline Estimated no. 2 & -0.0298 & 12.609 & -0.3065 & -161.54 & -1.5464 & 0.0013 & -7.9295 & -1.8485 & 0.1167 & -11.374 & -5.9544 \\
\hline
\end{tabular}

${ }^{a} S C D s$ were given by equation (33) where the flight velocity was $V=50 \mathrm{~m} / \mathrm{s}$.

${ }^{\mathrm{b}} \mathrm{SCD}$ were given by equation (33) where the flight velocity was $V=150 \mathrm{~m} / \mathrm{s}$.

\subsubsection{Polytopic model case}

Table 3 shows the initial and the estimated SCDs of the polytopic models, where 'no. 1' and 'no. 2' mean the first and the second model parameters, respectively. The initial SCDs were given as the values at $V=50$ and $150 \mathrm{~m} / \mathrm{s}$, respectively. The
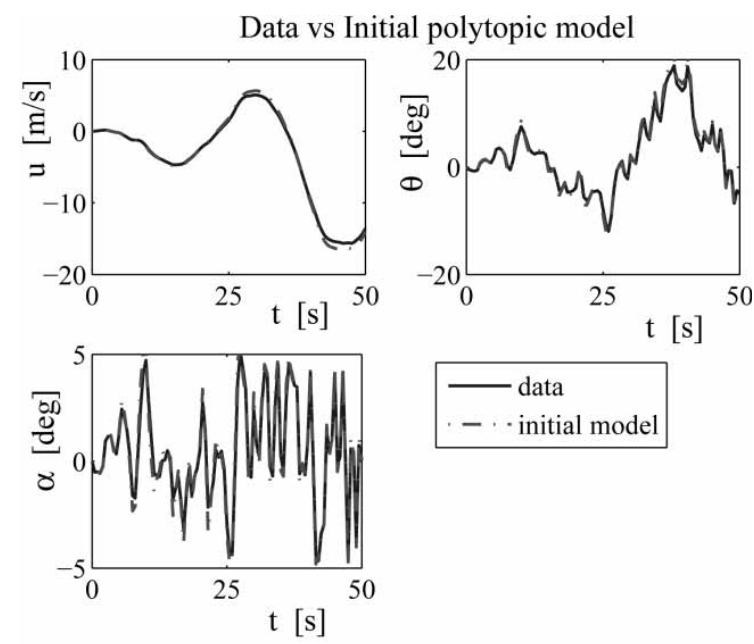

(a) Initial polytopic model.
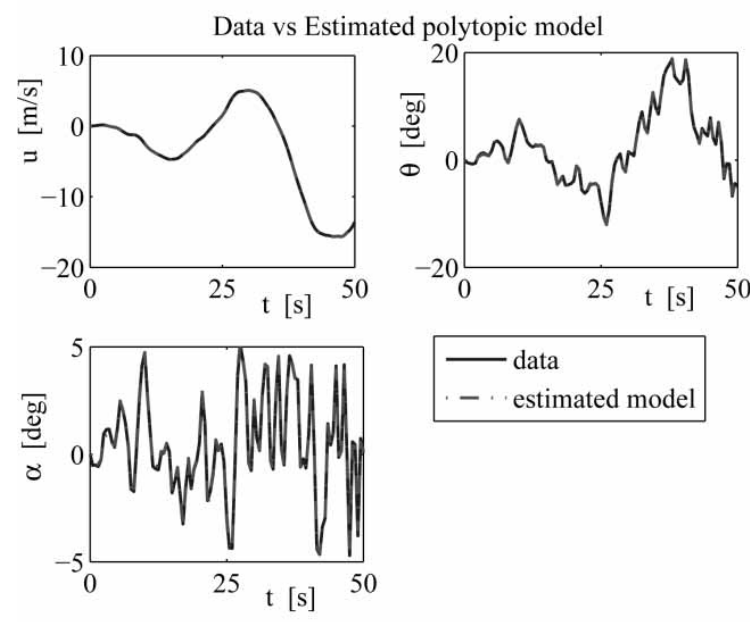

(b) Estimated polytopic model.

Fig. 4 Comparison between output data and outputs of initial and estimated polytopic models estimated SCDs were moved from the initial SCDs to the inside of the flight region. Figure 4 shows the output responses of the initial and the estimated polytopic models. The output of the estimated polytopic model was better fitted to the output data than that of the initial polytopic model. It was also seen in the r.m.s. as shown in Table 4.

Letting $P_{\text {poly }}(s, V)$ be the transfer function of the initial or the estimated polytopic model, Fig. 5 shows the plots of $\delta_{v}\left(P_{\text {lpv }}, P_{\text {poly }}\right)$, where the solidand the dashed-dotted lines indicate that $P_{\text {poly }}(s, V)$ is the estimated and the initial polytopic models, respectively. $\delta_{v}\left(P_{\mathrm{lpv}}, P_{\text {poly }}\right)$ whose $P_{\text {poly }}(s, V)$ was the estimated polytopic model was smaller than that whose $P_{\text {poly }}(s, V)$ was the initial polytopic model except near both edges of the flight region.

Summarizing the identification simulation, the polytopic model was more suitable for expressing

Table 4 R.m.s. of prediction error for each output channel of initial and estimated polytopic models

\begin{tabular}{llll}
\hline Model & $\boldsymbol{e}_{u}(\mathrm{~m} / \mathrm{s})$ & $\boldsymbol{e}_{\theta}\left({ }^{\circ}\right)$ & $\boldsymbol{e}_{\alpha}\left(^{\circ}\right)$ \\
\hline Initial & 0.4115 & 0.6847 & 0.4228 \\
Estimated & 0.0108 & 0.1255 & 0.1086 \\
\hline
\end{tabular}

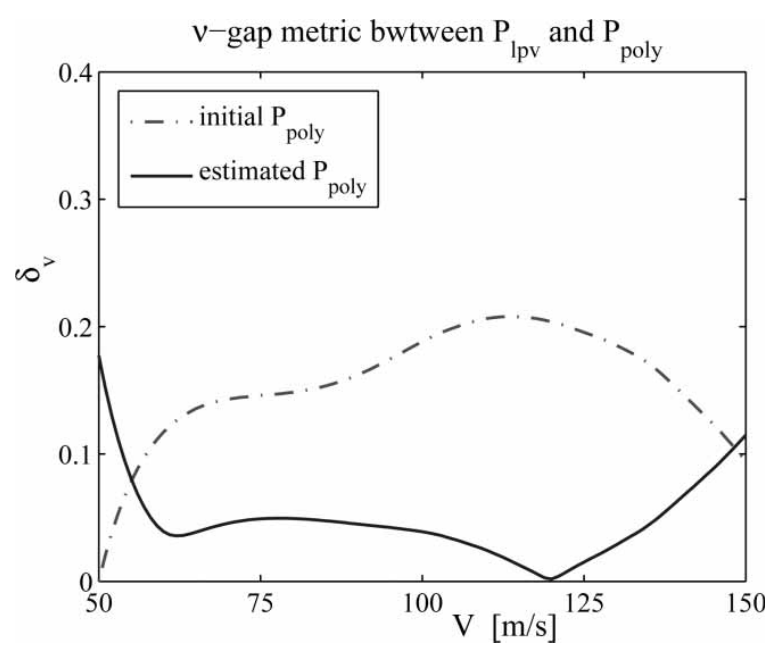

Fig. $5 v$-gap metric between $P_{\mathrm{lpv}}(s, V)$ and $P_{\text {poly }}(s, V)$ in the flight region, $50 \leqslant V(t) \leqslant 150 \mathrm{~m} / \mathrm{s}$ 
the behaviours of the LPV aircraft system than the LTI model from the viewpoints of the time and the frequency responses. Applying the prediction error method to the polytopic model, the parameters of the polytopic model were adjusted so as to fit the model output to the output data and make the model error small over the entire flight region.

\section{CONCLUDING REMARKS}

This article has presented a parameter estimation of continuous-time polytopic models for an LPV system. The prediction error method of LTI models was modified for polytopic models and was applied to an LPV aircraft system whose varying parameter was the flight velocity and estimation parameters were the SCDs. In an identification simulation, the polytopic model was more suitable for expressing the behaviours of the LPV aircraft system than the LTI model from the viewpoints of the time and the frequency responses. The SCDs of the initial polytopic model were adjusted so as to fit the model output to the output data obtained from the LPV aircraft system.

The presented technique may be applicable for polytopic models for non-linear systems, in which the reference trajectory is given in advance $[\mathbf{9}, \mathbf{1 0}]$. It should be needed to pay attention to the selection of the varying parameter. Multiple varying parameters may be required to express the non-linear system by a polytopic model. Furthermore, the number of operating points may be increased according to the change in the varying parameters. These will be examined in future works. In this article, the weighting functions of the polytopic model were assumed to be given in advance. Adjusting not only the model parameters but also the parameters of the weighting functions, the quality of the estimated polytopic model will be improved. This is also a future subject of research.

\section{REFERENCES}

1 Apkarian, P., Gahinet, P., and Becker, G. Self-scheduled $\mathcal{H}_{\infty}$ control of linear parameter-varying systems: a design example. Automatica, 1995, 31, 1251-1261.

2 Apkarian, P. and Adams, R. J. Advanced gain-scheduling techniques for uncertain systems. Advances in linear matrix inequality methods in control, 2000, pp. 209-248 (SIAM, Philadelphia).

3 Boyd, S., Ghaoui, L. E., Feron, E., and Balakrishnan, V. Linear matrix inequalities in system and control theory, 1994, vol. 15, (SIAM, Philadelphia).

4 Fujimori, A. Descriptor polytopic model of aircraft and gain scheduling state feedback control. Trans. Jap. Soc. Aeronaut. Space Sci., 2004, 47, 138-145.
5 Ljung, L. System identification-theory for the user, 2nd edition, 1999, (Prentice Hall, Upper Saddle River, New Jersey).

6 Vinnicombe, G. Uncertainty and feedback $\left(\mathcal{H}_{\infty}\right.$ loopshaping and the v-gap metric), 2001 (Imperial College Press, London).

7 Stilwell, D. J. State-space interpolation for a gainscheduled autopilot. J. Guid. Control Dyn., 2001, 24, 460-465.

8 Shin, J.-Y., Balas, G. J., and Kaya, M. A. Blending methodolgy of linear parameter varying control synthesis of F-16, aircraft system. J. Guid. Control Dyn., 2002, 25, 1040-1048.

9 Fujimori, A., Terui, F., and Nikiforuk, P. N. Flight control design of an unmanned space vehicle using gain scheduling. J. Guid. Control Dyn., 2005, 28, 96-105.

10 Fujimori, A., Gunnarsson, S., and Norrlöf, M. A gain scheduling control of nonlinear systems along a reference trajectory. Proceedings of CD-ROM of 16th IFAC World Congress, 2005, Th-A02-TP/1.

11 Boukhris, A., Mourot, G., and Ragot, J. Non-linear dynamic system identification: a multi-model approach. Int. J. Control, 1999, 75, 591-604.

12 Leith, D. J. and Leithead, W. E. Analytic framework for blended multiple model systems using linear local models. Int. J. Control, 1999, 75, 605-619.

13 Shorten, R., Murray-Smith, R., Bjorgan, R., and Gollee, H. On the interpretation of local models in blended multiple model structures. Int. J. Control, 1999, 75, 620-628.

14 McKelvey, T. and Helmersson, A. System identification using an over-parameterized model class - improving the optimization algorithm. Proceedings of the 35th IEEE Conference on Decision and control, 1997, pp. 2984-2989.

15 Verdult, V., Ljung, L., and Verhaegen, M. Identification of composite local linear state-space models using a projected gradient search. Int. J. Control, 2002, 75, 1385-1398.

16 Schmidt, L. V. Introduction to aircraft flight dynamics, 1998 (AIAA, Reston).

17 Isozaki, K., Masuda, K., Taniuchi, A., and Watari, M. Flight test evaluation of variable stability airplane (in Japanese). KHI. Tech. Rev., 1980, 75, 50-58.

\section{APPENDIX}

\section{Notation}

$a_{v}$

$\mathbf{A}_{i}, \mathbf{B}_{i}$,

$\mathbf{C}_{i}, \mathbf{D}_{i}$

$\mathbf{A}_{\mathrm{lpv}}, \mathbf{B}_{\mathrm{lpv}}$,

$\mathbf{C}_{\mathrm{lpv}}, \mathbf{D}_{\mathrm{lpv}}$

$\mathbf{A}_{\text {poly }}, \mathbf{B}_{\text {poly }}$

$\mathbf{C}_{\text {poly }}, \mathbf{D}_{\text {poly }}$

$\boldsymbol{e}$

$\boldsymbol{e}_{l}$ acceleration

system matrices of $i$ th local LTI model

system matrices of LPV system

system matrices of polytopic model

predicted error vector

vector whose $l$ th element is one and others are zeros 


\begin{tabular}{|c|c|c|c|}
\hline $\mathbf{F}, \mathbf{G}, \mathbf{C}, \mathbf{H}$ & system matrices of discrete-time & $X_{u}, X_{\alpha}$ & stability derivatives \\
\hline & $\begin{array}{l}\text { preaictor } \\
\text { system matrices of continuous-time }\end{array}$ & $\begin{array}{l}Z_{u}, Z_{\alpha}, \\
Z_{a}, M_{u}\end{array}$ & \\
\hline $\mathbf{C}_{\text {poly }}, \mathbf{H}_{\text {poly }}$ & predictor & $M_{\alpha}, M_{\dot{\alpha}}$ & \\
\hline & acceleration due to gravity & $M_{q}$ & \\
\hline$H_{N}$ & Hessian & $y$ & output vector of polytopic model \\
\hline$J_{N}$ & quadratic function & $\hat{\boldsymbol{y}}$ & predicted output vector \\
\hline$K_{\text {poly }}$ & filter gain of predictor & $\boldsymbol{y}_{\mathrm{lpv}}$ & output vector of LPV system \\
\hline $\boldsymbol{n}_{x}, \boldsymbol{n}_{y}, \boldsymbol{n}_{v}$ & $\begin{array}{l}\text { dimensions of state, output, and input } \\
\text { vectors }\end{array}$ & $\begin{array}{l}z \\
Z_{\delta_{e}}, M_{\delta_{e}}\end{array}$ & $\begin{array}{l}\triangleq\left[\begin{array}{ll}y_{\mathrm{lpv}}^{\mathrm{T}} & v^{\mathrm{T}}\end{array}\right]^{\mathrm{T}} \\
\text { control derivatives }\end{array}$ \\
\hline$N$ & number of sampled data & $\mathbf{Z}^{N}$ & data set \\
\hline$p$ & dimension of model parameter & & \\
\hline & vector & $\alpha$ & angle of attack \\
\hline$P_{\mathrm{lpv}}$ & transfer function of LPV system & $\delta$ & small positive value \\
\hline$P_{\mathrm{lti}}$ & transfer function of LTI model & $\delta_{\mathrm{e}}$ & elevator angle \\
\hline$P_{\text {poly }}$ & transfer function of polytopic model & $\delta_{v}$ & $\nu$-gap metric \\
\hline$q$ & pitch rate & $\eta$ & collected model parameter vector \\
\hline$r$ & number of operating points & $\boldsymbol{\eta}_{l}$ & $l$ th element of $\boldsymbol{\eta}$ \\
\hline$t$ & time & $\hat{\boldsymbol{\eta}}$ & estimate of collected model \\
\hline$T$ & sampling time & & barometer vector \\
\hline$u$ & forward velocity & $\theta$ & pitch angle \\
\hline$v$ & input vector & $\mu$ & step size \\
\hline$V$ & flight velocity & $\xi$ & model barometer vector \\
\hline$V_{1}, V_{2}$ & lower and upper flight velocities & $\xi_{i}$ & $i$ th model barometer vector \\
\hline$w_{i}$ & $i$ th weighting function & $\bar{\sigma}$ & maximum singular value \\
\hline$x$ & state vector of polytopic model & $\tau$ & varying parameter \\
\hline$\hat{\boldsymbol{x}}$ & predicted state vector & $\tau_{i}$ & $i$ th operating point \\
\hline$x_{\ln }$ & state vector of LPV system & & gradient with respect to $\boldsymbol{n}$ \\
\hline
\end{tabular}


Copyright of Proceedings of the Institution of Mechanical Engineers -- Part $G$-- Journal of Aerospace Engineering is the property of Professional Engineering Publishing and its content may not be copied or emailed to multiple sites or posted to a listserv without the copyright holder's express written permission. However, users may print, download, or email articles for individual use. 


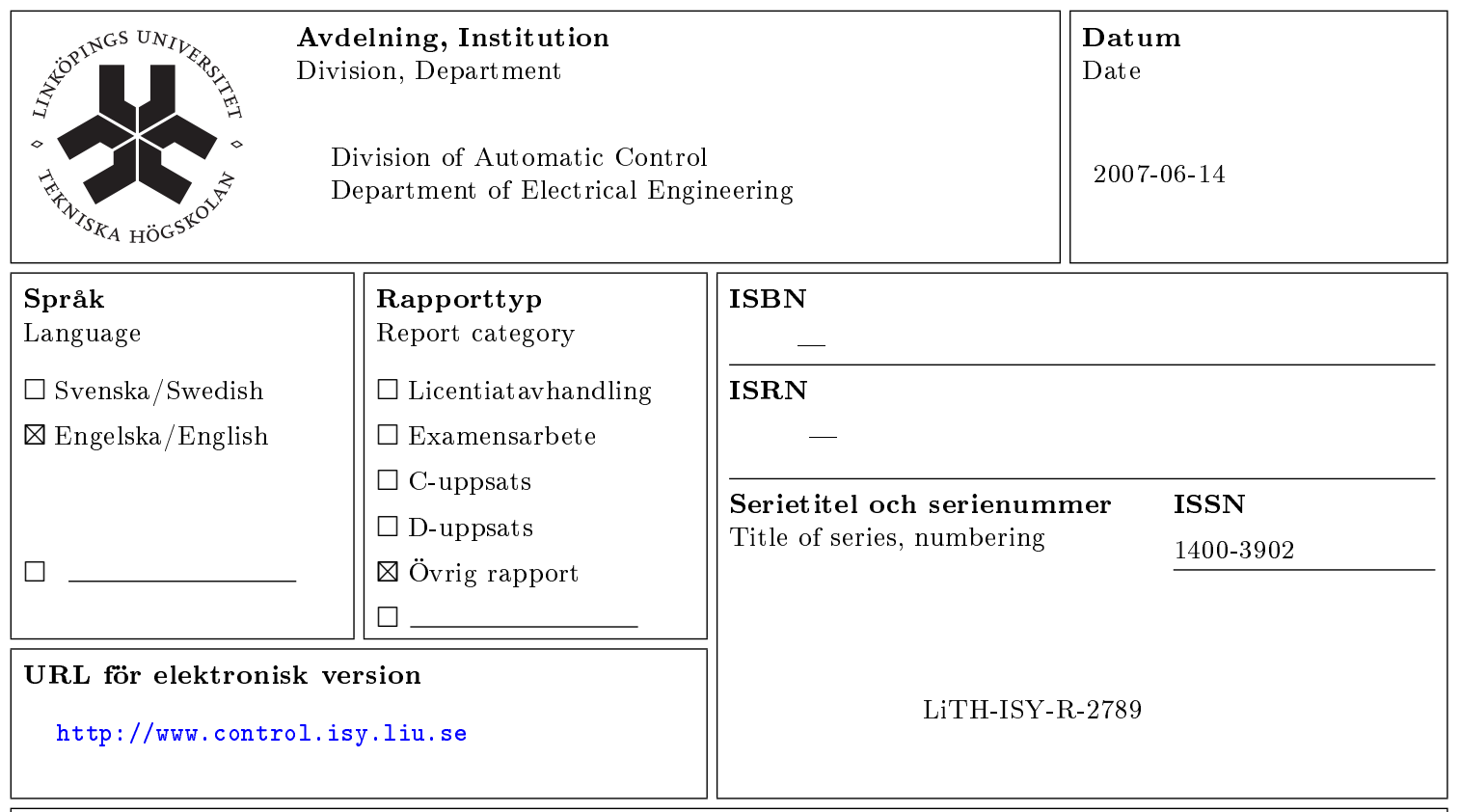

Titel Model identification of linear parameter varying aircraft systems

Title

Författare Atsushi Fujimori, Lennart Ljung

Author

\section{Sammanfattning}

Abstract

This article presents parameter estimation of continuous-time polytopic models for a linear parameter varying (LPV) system. The prediction error method of linear time invariant (LTI) models is modified for polytopic models. The modified prediction error method is applied to an LPV aircraft system whose varying parameter is the flight velocity and model parameters are the stability and control derivatives (SCDs). In an identification simulation, the polytopic model is more suitable for expressing the behaviours of the LPV aircraft than the LTI model regarding time and frequency responses. The SCDs of the initial polytopic model are adjusted to fit the model output dta obtained from the LPV aircraft system. 